\title{
SIMPLICIAL INTERSECTION CHAINS FOR AN ABSTRACT COMPLEX
}

\author{
W. W. FLEXNER
}

Abstract complexes have been defined by J. W. Alexander, W. Mayer, A. W. Tucker and S. Lefschetz. ${ }^{1}$ The definition here adopted is that of Lefschetz which derives directly from that of Tucker. To conform to Lefschetz's present usage the notation here will differ from that in the article cited by having $K=\left\{E_{i}^{p}\right\}$ and $K^{*}=\left\{E_{p}^{i}\right\}$. It should be remarked that when $K$ is infinite $F F=0 \mathrm{im}$ plies that for given $E_{t}^{p}$ and $E_{j}^{p-2}$,

$$
\left[E_{i}^{p}: E_{k}^{p-1}\right]\left[E_{\mathfrak{k}}^{p-1}: E_{\jmath}^{p-2}\right] \neq 0
$$

for at most a finite number of $E_{k}^{p-1}$. The dual $K^{*}$ here is to have the property

$$
\left[E_{i}^{p}: E_{j}^{p-1}\right]=(-1)^{p+1}\left[E_{p-1}^{j}: E_{p}^{i}\right]=\mu_{i j}^{p}
$$

where $\mu_{i j}^{p}$ is an abbreviated notation (compare loc. cit., p. 346 (c)). This is in order that a fourth postulate

$$
\text { IV. } \mathrm{Ki}\left(E_{i}^{p} \cdot E_{p}^{b}\right)=1
$$

may be added to I-III (loc. cit., p. 350), where Ki means Kronecker index, the sum of the coefficients of the elements of the zero-chain in question. Here $C$ is a $p$-chain of $K, D$ a $q$-chain of $K^{*}, p>q$.

It is well known that when $K$ is a simplicial or polyhedral manifold, for every pair $E_{t}^{p}, E_{q}^{j}, p \geqq q$, an intersection can be defined which is a chain of the simplicial regular subdivision of $K$. And when $K$ is an arbitrary simplicial complex, the intersection is on $K$ and hence simplicial. Here it is shown that for any complex the intersections $E_{i}^{p} \cdot E_{q}^{j}$ can be regarded as integral chains of a simplicial complex $K^{\pi}$.

This complex will be defined abstractly by means of its vertices $\sigma_{i}^{p}$. The first definition is for $p=q$ :

$$
E_{i}^{p} \cdot E_{p}^{j}=\delta_{i}^{j} \sigma_{j}^{p}
$$

(where - replaces the $\odot$ of the article cited). Then for $p>q$, if $r=p-q-1$,

$$
E_{i}^{p} \cdot E_{q}^{j}=\sum_{k_{1}, k_{2}, \cdots, k_{r}} \stackrel{p}{\mu_{i k_{1}} \mu_{k_{1} k_{2}}^{p-1}} \cdots \stackrel{\mu_{k_{r} j}^{q+1} \sigma_{i} \sigma_{k_{1}}^{p-1}}{q+1} \cdots \sigma_{k_{r} \sigma_{j}}^{q+1} \sigma_{j}^{q}
$$

${ }^{1}$ S. Lefschetz, this Bulletin, vol. 43 (1937), pp. 345-359. (References to the other authors will be found on page 345 .) 
where $\sigma_{i}^{p} \sigma_{k_{1}}^{p-1} \cdots \sigma_{k_{r}}^{q+1} \sigma_{j}^{q}$ denotes the simplex of $K^{\pi}$ with the given vertices; so $E_{i}^{p} \cdot E_{q}^{j}$ is an integral chain of $K^{\pi}$.

To verify that $E_{i}^{p} \cdot E_{q}^{j}$ is an intersection, it is sufficient, in view of (1), (2), to prove the formula III (p. 350, loc. cit.):

III. $F\left(E_{i}^{p} \cdot E_{q}^{j}\right)=\left(F E_{i}^{p}\right) \cdot E_{q}^{j}+(-1)^{p} E_{i}^{p} \cdot\left(F E_{q}^{j}\right)$.

When $q=p-1, r=0$, (2) gives $E_{i}^{p} \cdot E_{p-1}^{j}=\mu_{i j}^{p} \sigma_{i}^{p} \sigma_{j}^{p-1}$. Using the rule for the boundary of a simplex given by its vertices, we obtain $F\left(E_{i}^{p} \cdot E_{p-1}^{j}\right)=\mu_{i j}^{p}\left(\sigma_{j}^{p-1}-\sigma_{i}^{p}\right)$. By formula (2) (loc. cit.), its dual, and (1),

$$
\begin{aligned}
& \left(F E_{i}^{p}\right) \cdot E_{p-1}^{j}=\sum_{k} \underset{\mu_{i k}}{p} E_{k}^{p-1} \cdot E_{p-1}^{j}=\stackrel{p}{\mu_{i j} \sigma_{j}^{p-1}}, \\
& E_{i}^{p} \cdot\left(F E_{p-1}^{j}\right)=(-1)^{p+1} \mu_{i j}^{p} \sigma_{i}^{p}
\end{aligned}
$$

which verifies III in this case. When $q<p-1$,

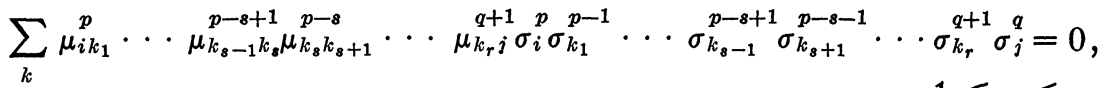

$$
\begin{aligned}
& 1 \leqq s \leqq r \text {, }
\end{aligned}
$$

since $F F=0$ implies $\sum_{k_{s}} \mu_{\mathbb{k}_{s-1} k_{s}}^{p-s+1} \mu_{\mathbb{k}_{s} k_{s+1}}^{p-s}=0$. Hence

$$
\begin{aligned}
& F\left(E_{i}^{p} \cdot E_{q}^{j}\right)=\sum_{k} \stackrel{p}{\mu_{i k_{1}}} \cdots \mu_{k_{r} j}^{q+1}\left(\sigma_{k_{1}}^{p-1} \cdots \sigma_{k_{r}}^{q+1} \sigma_{j}^{q}\right. \\
& \left.+(-1)^{p-q} \sigma_{i}^{p} \sigma_{k_{1}}^{p-1} \cdots \sigma_{k_{r}}^{q+1}\right) \\
& =\sum_{k_{1}}{ }_{\mu_{i k_{1}}}^{p}\left(E_{k_{1}}^{p-1} \cdot E_{q}^{j}\right)+(-1)^{p-q} \sum_{k_{r}} \mu_{k_{r} j}^{q+1}\left(E_{i}^{p} \cdot E_{q+1}^{k_{r}}\right)
\end{aligned}
$$

which by Lefschetz's article, formulas (2) and (1), is the right side of III.

Cornell University and

Institute for Advanced Study 